\title{
The Factual Data on the Ceselestial Bodies Influences on Seismic Activity
}

\author{
Sergey A. Vasiliev ${ }^{1} \&$ Virginia Nina Tataridou $^{1}$ \\ ${ }^{1}$ Scientific Research Institute of Exploration Geophysics VNIIGeofizika (retired), Moscow, Russia \\ Correspondence: Sergey A. Vasiliev, 38 Nazliu Str., Palio Faliro, Athens 17564, Greece. Tel: 30-210-948-0145. \\ E-mail: disput22@gmail.com,disput22@mail.ru
}

Received: November 21, 2012 Accepted: December 17, 2012 Online Published: January 5, 2013

doi:10.5539/apr.v5n1p36

URL: http://dx.doi.org/10.5539/apr.v5n1p36

\begin{abstract}
The factual data on the influences of the Sun, Moon, planets, star on seismic activity are described. In the paper, the influences of the planet Mars and the Sun on seismic activity are confirmed statistically reliably in the subregion of the U.S. west coast and part of Mexico, in the subregion of Japan and in the world as a whole. The common properties and certain differences of the actions of the Mars and the Sun on seismic activity are marked. In particular, the obtained statistical data confirm that, in some cases, the Mars actions on seismic activity appear more strongly of the Sun actions. Within the framework of the classical (non-quantum) field theory, these data imply the existence of the long-range action fields (of LRA-fields). Previously, the authors developed a physical model of LRA-fields as the logical consequence of the nonseismological experimental and observational data. This model reveals the structure, specific properties and the origin conditions of LRA-fields. The physical model of LRA-fields predicts and allows to explain correctly the specific features of the actions of the planets and other celestial bodies on seismic activity. Monitoring of LRA-fields opens, in principle, new perspectives of the seismic activity and solar weather forecasting, new perspectives of tracking of the Earth and celestial bodies interior processes changes since the variations of LRA-fields reflect the changes of the internal processes of their sources.
\end{abstract}

Keywords: seismic activity, celestial bodies, planets influences, actions, earthquakes, long-range action fields, LRA-fields, energy-free

\section{Introduction}

It is hard to imagine that solar system planets and even more so stars may influence on seismic activity, in addition, may influence significantly. However, according to the factual data, the above influences are real together with the influences of the Sun and Moon (see Sections 2 and 3 of this paper). Moreover, in some cases, the planets influences on seismic activity appear more strongly of the Sun influence (Sections 2 and 3). But, according to astrophysics, the planets cannot influence on seismic activity. Astrophysics firmly states one's position: the planets (even more so stars) are unable to influence on the Earth. This position has the following substantiation in astrophysics.

Substantiation. Actually, the total energy flow of a field (known or still unknown to us) through its frontal area must be constant and must be spread throughout the frontal area. The frontal area increases with respect to $r^{2}$ (in the case of its spherical shape, where $r$ is the distance from the point-source of the field). Finally, the energy-flux density of the field together with the field intensity should decrease with respect to $1 / r^{2}$ or faster. The corresponding numerical estimates lead astrophysics to the present position.

Thus, the paradox arises: on the one hand, according to the substantiation, these influences can not exist, on the other hand they exist in reality. Whether it is possible to solve the paradox and to give an explanation to the paradoxical influences on seismic activity? This problem is considered in Section 4.

\section{The Factual Data}

The influence of the Sun on seismic activity has been investigated in seismology in detail. The actions of the Sun are considered in seismology as a trigger hook of earthquakes (trigger effect), but not as a reason of an energy pumping to the earthquakes preparation zone. In seismology the year cycle of seismic activity is known. The changes of the earthquakes frequency on months during the year cycle stably reach tens percents in the relatively small seismically active subregions (Levin et al., 2005; Sasorova et al., 2006; Andreeva et al., 2009). At that, the 
distributions of earthquakes on months are so different in the different subregions, that in the large region - South America - the distribution of earthquakes on months is considerably smoothed (Sasorova et al., 2006). Hence, the Sun acts on the seasonal changes of seismic activity essentially differently in different subregions. Several years ago the information have appeared about the statistically significant diurnal periodicity of earthquakes from representative sections of the earthquakes catalogues (Sidorin, 2009, here we are talking about solar days). According to Sidorin (2009), the version about essential influences of the tidal effects on seismicity is not confirmed.

The versions about the actions on seismicity of the mechanical loading seasonal changes on the Earth's surface (changes of atmospheric pressure, the weight of ice and snow cover, etc.) and seasonal changes in the ionosphere (via the action of its electromagnetic field) exist. Seasonal changes in the mechanical loading occurring in an antiphase in the Northern and Southern hemispheres. However, the same antiphase variations in seismic activity was not detected. The information about the solar activity influences on the time earthquakes series was published (Khain et al., 2008). The reasons for the solar activity influences was not established, although, of course, there are assumptions related to the electromagnetic fields actions.

As a whole, Magnitsky's estimation remains valid (Magnitsky, 2006): “Although earthquakes are caused by Earth's tectonic activity, nevertheless, numerous endeavours to establish a relationship between these and other phenomena are made. Now relationship of earthquakes number with the Earth year revolution around the Sun deserves the most attention. Of all the other dependences this relationship is statistically manifested with the most reliability. However the nature of such relationship, if it really exist, remains completely vague".

At similar examinations, it is better to accumulate the earthquakes quantity not on calendar days (they are shifted relative to the sidereal year), but on the degrees of the $\varphi$ ecliptic longitude of the Sun or planet. The $\varphi$ ecliptic longitude is the ecliptic degree of the celestial body position measured from the $\gamma$ vernal equinox point in the direction of the Sun motion on the ecliptic. What is the physical sense of the $\varphi$ ecliptic longitude when the Sun and planets are located in the ecliptic plane? Let $\mathbf{R}_{\mathrm{E}}$ is the vector drawn from the Sun or planet to Earth. The direction of the $\mathbf{R}_{\mathrm{E}}$ vector varies at motions of the Earth and planets around of the Sun. The displacement of the Sun or a planet by one degree on the $\varphi$ ecliptic longitude corresponds to a rotational displacement of the $\mathbf{R}_{\mathrm{E}}$ vector also on one degree. Let $\psi$ is the $\mathbf{R}_{\mathrm{E}}$ vector rotation angle. In view of this, at suitable choice of the $\psi$ angle computing origin, $\varphi$ and $\psi$ angles are equal, $\varphi=\psi$. In other words, the ecliptic $\varphi$ longitude describes the $\psi$ angle of the $\mathbf{R}_{\mathrm{E}}$ vector rotation. The suitable choice of the $\psi$ angle computing origin is: $\psi=0$ at such direction of $\mathbf{R}_{\mathrm{E}}$ vector when $\mathbf{R}_{\mathrm{E}}$ vector lies on the straight line between the Earth and the $\gamma$ vernal equinox point, i.e. when the Sun or planet is projected from the Earth by this line to the vernal (no autumn) equinox point. Everywhere below we will consider $\varphi=\psi$. If the planet or the Sun is the source of some physical field, the $\varphi$ ecliptic longitude describes the $\psi$ angular displacement of the Earth into this field at the movements of the Earth and the planets around the Sun. Although as a result of these movements, in the case of any solar system planet, the $\mathbf{R}_{\mathrm{E}}$ vector direction changes in complex fashion, the $\mathbf{R}_{\mathrm{E}}$ vector describes the finally full circle on $\psi$ angle from 0 to 360 degrees, i.e. in result the Earth is experiencing any solar system planet field action on all directions from the planet (in the ecliptic plane).

The following facts are most unintelligible. Nearly thirty years ago, Meidav and Sadeh discovered the action of pulsar CP1133 on seismicity (Sadeh et al., 1972). According to Weber, the energy of the pulsar gravitational waves is many orders of magnitude lower than that required for the detected pulsar effect on seismicity (Sadeh et al., 1972). At about the same time, Ben-Menachem, the famous seismologist, detected a correlation between seismicity and sunrises-sunsets that could not be explained.

If You ask seismologist - is there now the method of short-term (before a few days) forecast simultaneously of the location, time and magnitude of strong earthquakes which is vindicated with probability at least about 50 percent? - in most cases You get the answer - no, it is not exists. However, such innovative method exists and successfully passed the five-year practical testing in the Kamchatka Peninsula subregion that is little known. This is the method of Lezdinsh (2008). In result of his eighteen-year research Lezdinsh revealed correlation between earthquakes and positions of the planets, Sun and Moon relative to the Earth and the local horizon plane on the Kamchatka Peninsula subregion. On this (together with the seismological data monitoring) his method is based (Lezdinsh, 2008). Lezdinsh's method effectiveness proves influences of the celestials bodies, including the planets, on seismic activity. This proof is difficult to refute. According to Lezdinsh's practical data, action of each celestial body to seismic activity strongly depends on its position on ecliptic, that is, depends on its $\varphi$ ecliptic longitude. At that, the influences of the different celestial bodies are essentially different both on the action force, and on character of the action dependence on ecliptic longitude of the celestial body. For example, according to Lezdinsh's practical data, Mars, acts on seismic activity much more strongly the Sun. 
In Lezdinsh's method the following plays essential role. At the moment of each earthquake, each the planet, Sun and Moon have a certain locations (ecliptic longitude) on the ecliptic. Lezdinsh calculates these locations for each earthquake on Kamchatka. Only the representative section of the long-term earthquakes catalogue is taken into account. These locations are superimposed on the ecliptic. Lezdinsh found out pronounced, statistically significant concentrations of these locations for particular celestial bodies and more feebly marked concentrations for other celestial bodies. The last speaks about different "force" of different celestial bodies actions on seismic activity. The indicated locations concentration on the ecliptic and the celestial bodies actions "force" estimation are used then for the strong earthquakes forecast. By other words, according to the statistics and Lezdinsh's practical experience, the particular locations on the ecliptic of each celestial body either promote, or hinder to the earthquakes development. Such celestial bodies actions to earthquakes should receive confirmation by the seismic activity statistical examinations. And so it happens.

In the paper (Kiladze et al., 2005) statistically significant maxima and minima of earthquakes frequency are disclosed in the Caucasian subregion in depending on ecliptic longitude of the planets and on their relative positions. But in the paper (Kiladze et al., 2005) the nonuniformities of the planets motions on the ecliptic are not taken into account (at a look from the Earth). These nonuniformities are so significant that they are capable to levels the maxima and minima of the statistical dependences, even to convert maxima to minima and on the contrary. In the paper (Kiladze et al., 2005) the aftershocks distorting influence are not taken into account also. Meanwhile, a single strong earthquake with hundreds aftershocks in the representative earthquakes catalogue section is capable to give the pronounced maximum of the statistical diagram giving the false representation on the maximum of the celestial bodies action at their particular locations on the ecliptic. (The results of our test statistical processing of the earthquakes time series demonstrated such consequences of the not taking into account the influences of the nonuniformities and aftershocks in several seismoactive subregions.) Lezdinsha's works and the paper (Kiladze et al., 2005) stimulated the authors of present paper to have realized new statistical examinations of earthquakes frequency in various subregions and on all globe in dependence on the celestial bodies ecliptic longitude taking into account the nonuniformities of the celestial bodies motions on the ecliptic and taking into account the necessity to filter out aftershocks.

\section{The New Statistical Data on the Sun and Mars Influences on Seismic Activity}

Investigating earthquakes during the period 1973 - 2009 years, the authors found variations of the average earthquakes frequency depend upon the Mars ecliptic longitude (Figures 1, 2, 3 below) up to $26 \%$ in Region 1 (U.S West Coast and part of Mexico, the latitude range $25 \mathrm{~N}-45 \mathrm{~N}$, the longitude range $105 \mathrm{~W}-135 \mathrm{~W}$ ), up to $24 \%$ in Region 2 (Japan, the latitude range $28 \mathrm{~N}-47 \mathrm{~N}$, longitude range 126W - 150W) and up to 8\% in Region 3 (all terrestrial globe). The input data source was the catalog NEIC, total 12293 earthquakes in Region 1 with magnitude $M \geq 3.4,8689$ earthquakes in Region 2 with magnitude $M \geq 4.7$, and 58749 earthquakes in Region 3 with magnitude $M \geq 5$.

The $v_{k}$ frequency variations depending upon the Sun ecliptic longitude was confirmed also in the same regions in limits up to $18 \%$ (Region 1), up to $13 \%$ (Region 2) and up to $10 \%$ (Region 3) - figures 4, 5, 6 below.

As mentioned, according to Lezdinsh's data, Mars action to seismic activity on the Kamchatka Peninsula subregion essentially exceeds the Sun action. As you can see, the same is confirmed in regions 1 and 2 . At that, in each region 1 and 2 the actions of the Sun and Mars depend on celestial body ecliptic longitude significantly differently that is co-ordinated with the mentioned Lezdinsh data.

In the earthquakes time series processing the following was taken into account: changes of the Sun and Mars velocity on the ecliptic, the differences of the earthquakes catalog representation limits in different regions, the necessity to exclude aftershocks and so on. The ecliptic was divided into the equal $E_{k}$ segments, $=1,2, \ldots, 12$. The $k$ numbers of the segments increase in the Sun movement direction on ecliptic. The $E_{1}$ first segment begins and the $E_{12}$ last segment finishes in $\gamma$ vernal equinox point.

Let's designate as $N_{k j}$ the number of the earthquakes which has occurred in time of $j$-th passing of the celestial body through $E_{k}$ segment, $=1,2,3, \ldots$. The $N_{k}$ summarized number of earthquakes which has occurred during the repeated passing of the celestial body over $E_{k}$ segment in the period of time 1973-2009 years was calculated (accumulated) for each $E_{k}$ segment, that is, the $N_{k j}$ numbers were summarized on the $j$ index. (Only the integer number of the celestial body periods of revolutions on ecliptic was taken into account.) For the same period the $T_{k}$ summarized time of the celestial body location into $E_{k}$ segment was calculated. The $v_{k}$ average earthquakes frequency was determined by rule $v_{k}=N_{k} / T_{k}$. In other words, we used here the epoch superposition method to extract the celestial body influence from many significant influences on seismic activity (see Section 5). 


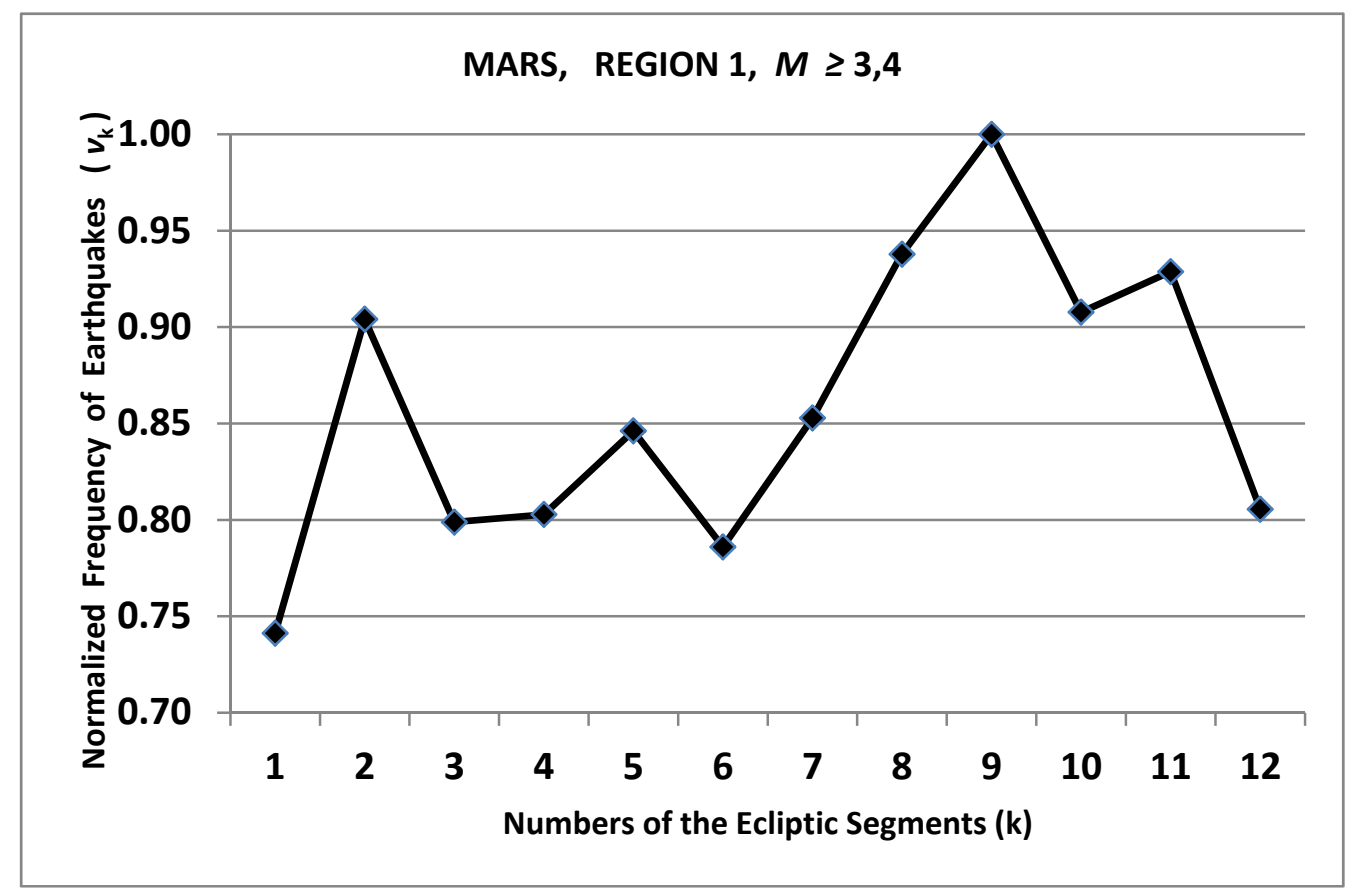

Figure 1. Region 1 - U.S. West Coast and part of Mexico. The $v_{k}$ variations of the average earthquakes frequency in depending on the Mars position on the ecliptic during the period 1973 - 2009, magnitude $M \geq 3.4$. The graph maximum normalized per unit

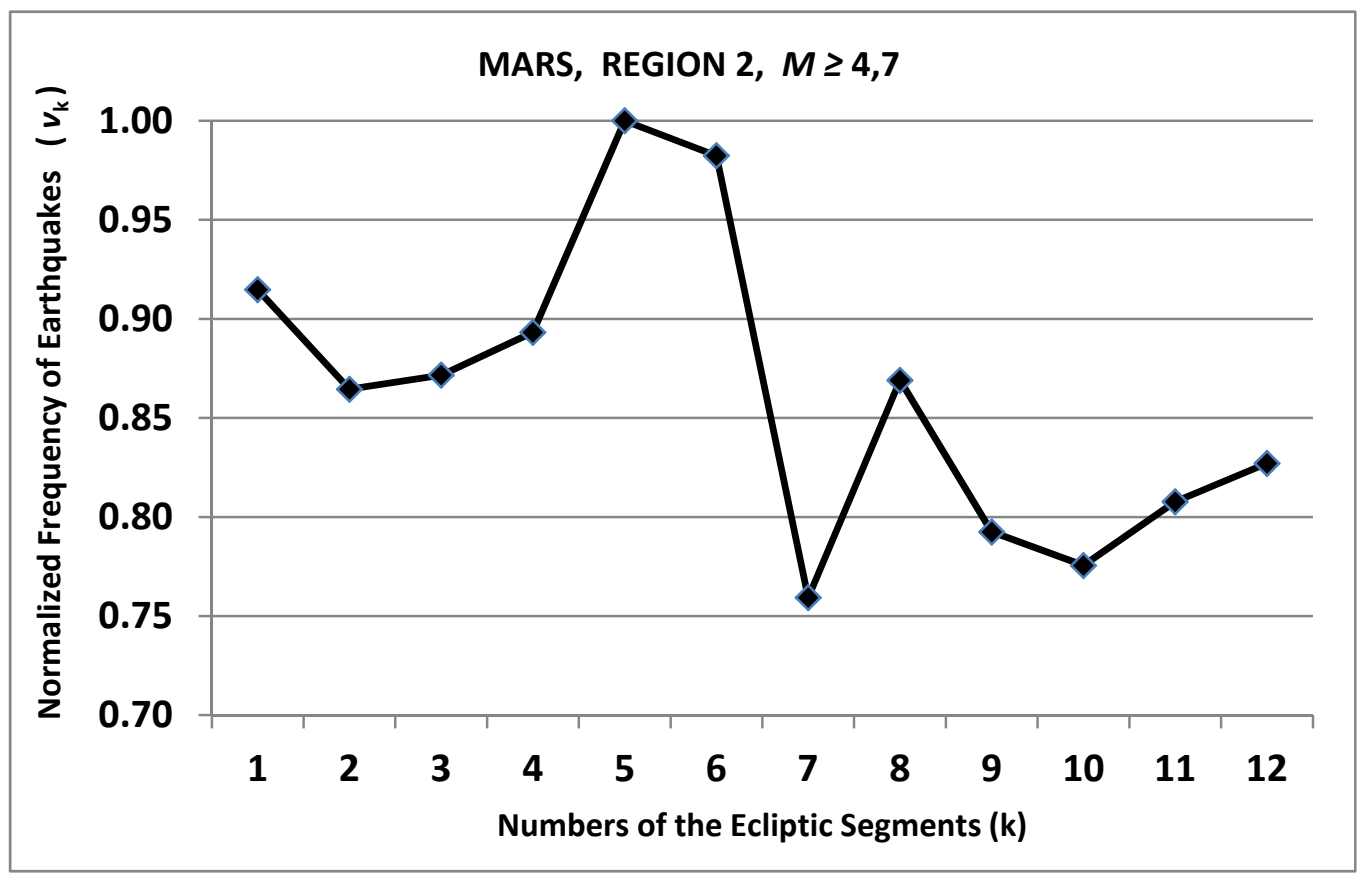

Figure 2. Region 2 - Japan. The variations of the $v_{k}$ average earthquakes frequency in depending on the Mars position on the ecliptic during the period $1973-2009$, magnitude $M \geq 4.7$. The graph maximum normalized per unit 


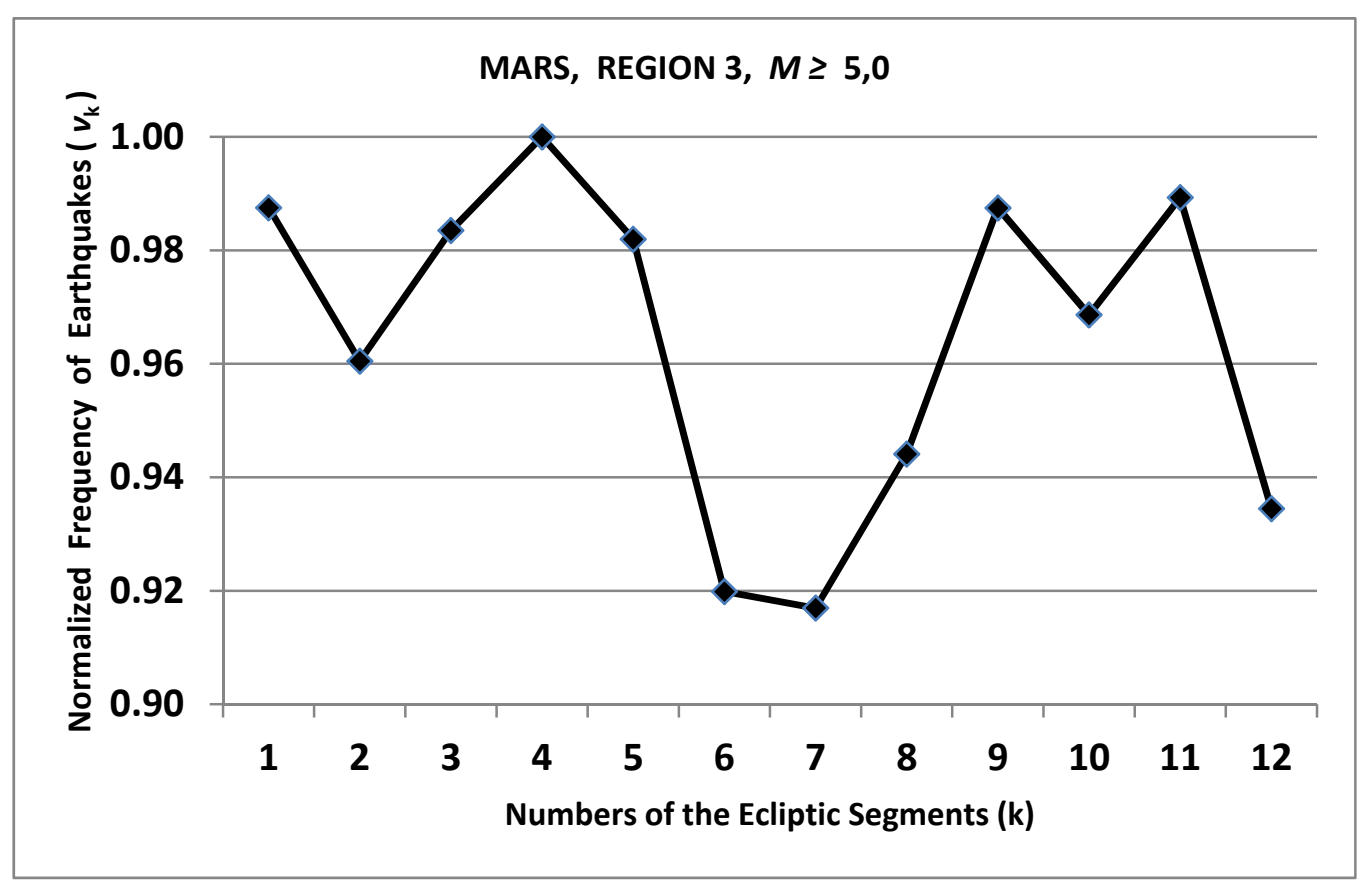

Figure 3. Region 3 - all terrestrial globe. The variations of the $v_{k}$ average earthquakes frequency in depending on the Mars position on the ecliptic during the period $1973-2009$, magnitude $M \geq 5$. The graph maximum normalized per unit

As it is simple to be convinced, the graphs for the Sun, approximately correspond to the earthquakes frequency alterations on months if months to count from the day of vernal equinox. For convenience of comparisons, the graphs maxima was normalized per unit. The mentioned above minimal magnitude values in each region correspond to the boundaries of the earthquakes catalogue representative section in these regions. At the estimations of the probability of the abmodality by formula Poisson, as it reasonably make in seismology (Vikulin, 2003, Wiemer, 2000, Yiğiter, 2012) after elimination of aftershocks, the main maximum and minimum on the figure 1 graph for Mars (region 1) are not random with the $P_{M A X}=0.99992$ and $P_{M I N}=0.9998$ probabilities, accordingly. For the Sun the same results (Figure 4) are not random in region 1 with the $P_{M A X}=$ 0.9989 and $P_{M I N}=0.9964$ probabilities. Practically the same values of $P_{M A X}, P_{M I N}$ probabilities are obtained for Mars in region 2. For the Sun $P_{M A X}=0.9898$ and $P_{M I N}=0.9972$ in region 2. In the consent with papers (Levin et al., 2005, Sasorova et al., 2006, Andreeva et al., 2009), the $v_{k}$ frequency graphs essentially vary, the maxima and minima are displaced at the passage from region 1 to region 2 - figures 1,2 and 4, 5. Hence, at the average of earthquakes frequency on all globe - region 3 - the $v_{k}$ frequency curves should be aligned essentially. It confirms the graphs of Figures 3 and 6 . Here $v_{k}$ frequency variations for Mars and the Sun are reduced approximately up to $8 \%$ and $10 \%$, accordingly. Despite of the essential reduction of the amplitude variations, their $P_{M A X}, P_{M I N}$ probabilities of nonrandomness remain enough high. In region 3, for the Sun $P_{M I N}=0.99998$, $P_{M A X}=0.99992$, for Mars $P_{M I N}=0.99997, P_{M A X}=0.9998$. It happens because of the common earthquakes number increases up the order at the passage to region 3.

\section{Analysis of the Factual Data from The Viewpoint of Classical Field Theory}

\subsection{The Paradox of the Planets Influences on Seismic Activity}

Thus, the relation between the vitally important phenomena - earthquakes - and the influences of the celestial bodies (including planets and stars) is disclosed. Concerning the Sun influences, the nature of such relation remains vague. The reasons of the year and diurnal (solar day) cycles of seismic activity and the reason of the solar and seismic activities relation are not ascertained though there are many hypotheses (Section 2) which quantity builds up. Concerning the planets influences we come to the paradox. The astrophysics negates such relation. According to astrophysics, the planets actions on seismic activity cannot be explained by trivial reasons - by the actions of electromagnetic and gravitational fields of the planets, by the tides caused by the planets, etc. (Section 1). But these planets influences exist. Accordingly, some planets actions properties are disclosed, but the 


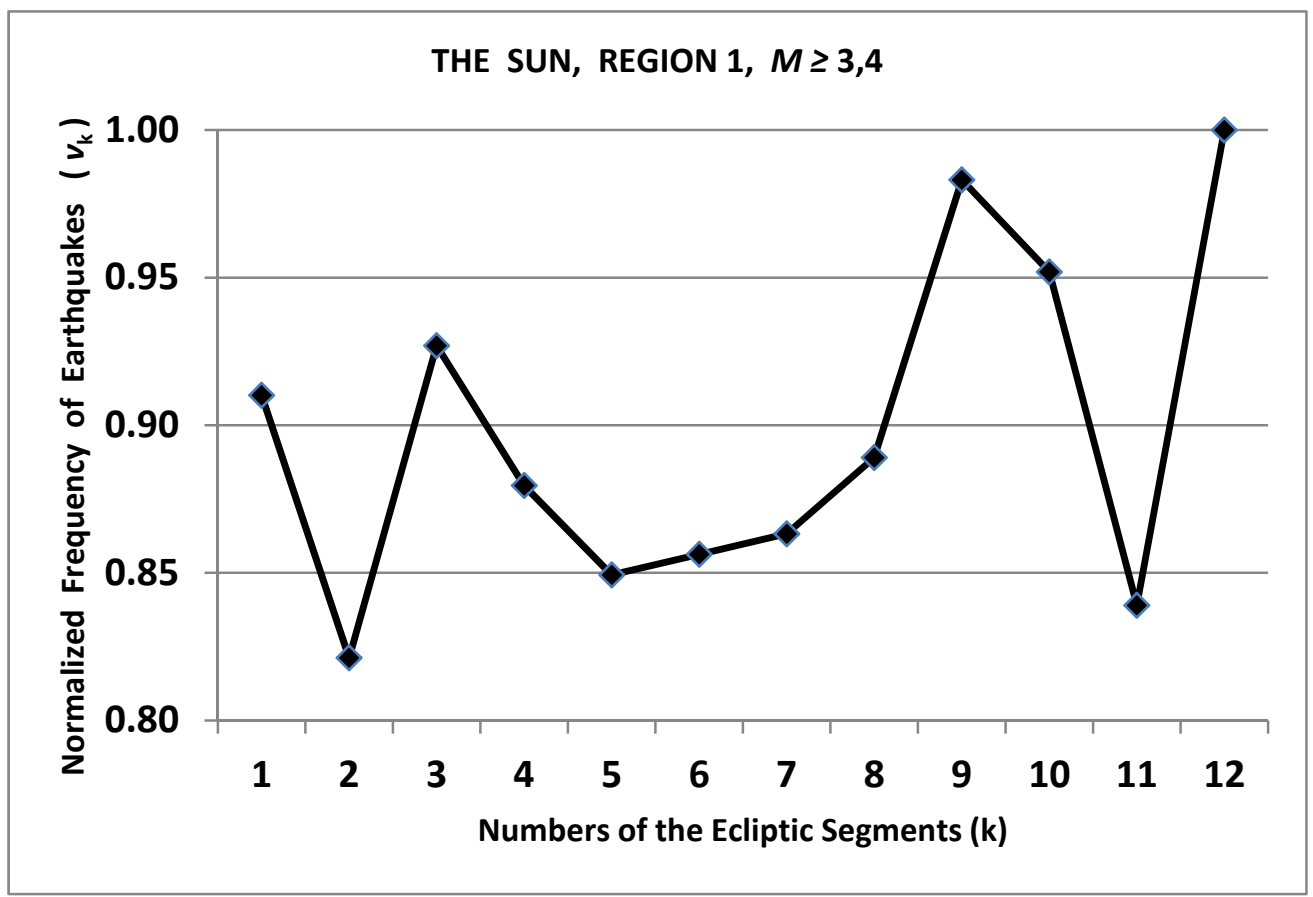

Figure 4. Region 1 - U.S. West Coast and part of Mexico. The variations of the $v_{k}$ average earthquakes frequency in depending on the Sun position on the ecliptic during the period $1973-2009$, magnitude $M \geq 3.4$. The graph maximum normalized per unit

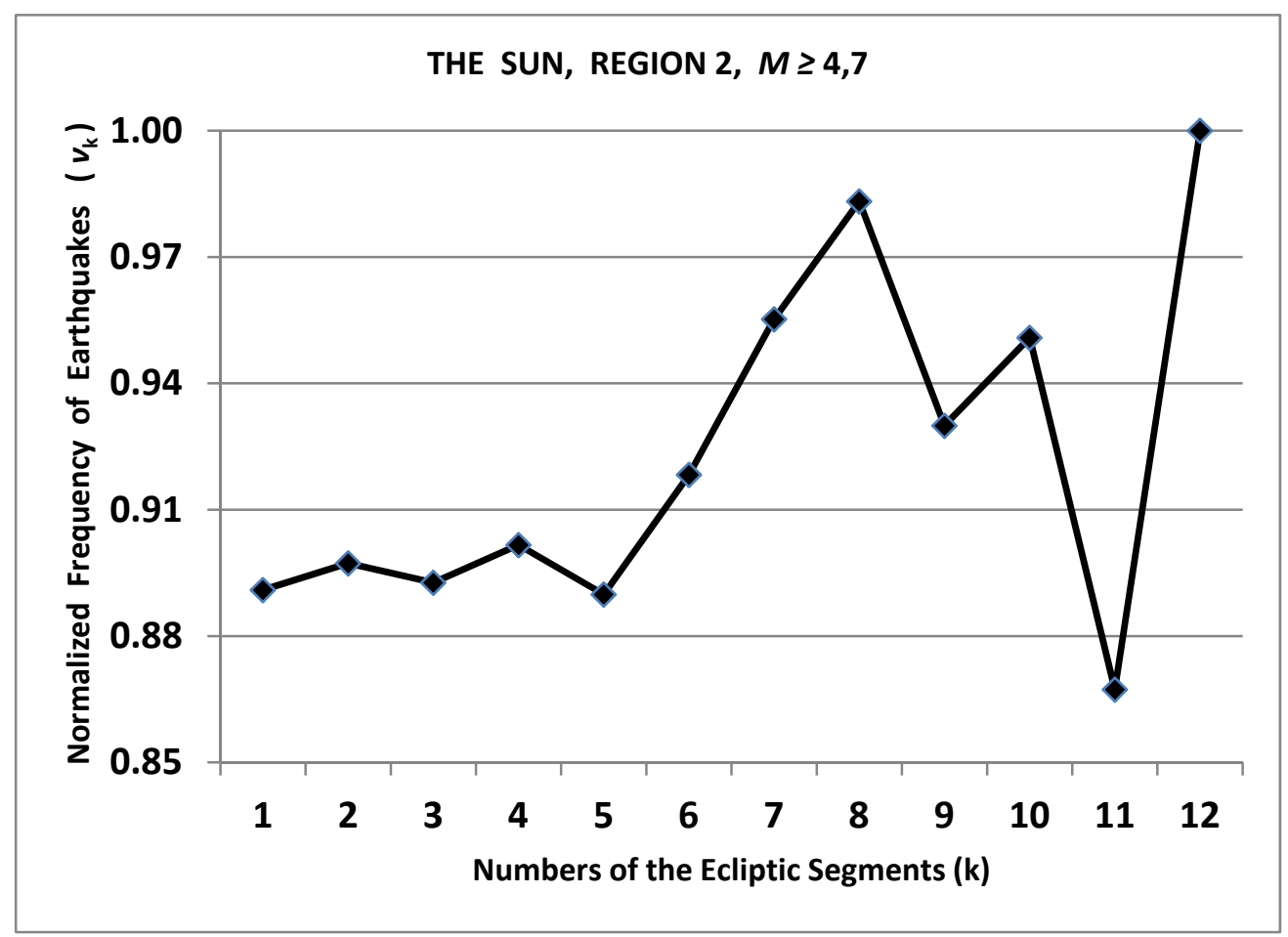

Figure 5. Region 2 - Japan. The variations of the $v_{k}$ average earthquakes frequency in depending on the Sun position on the ecliptic during the period 1973 - 2009, magnitude $M \geq 4.7$. The graph maximum normalized per unit 


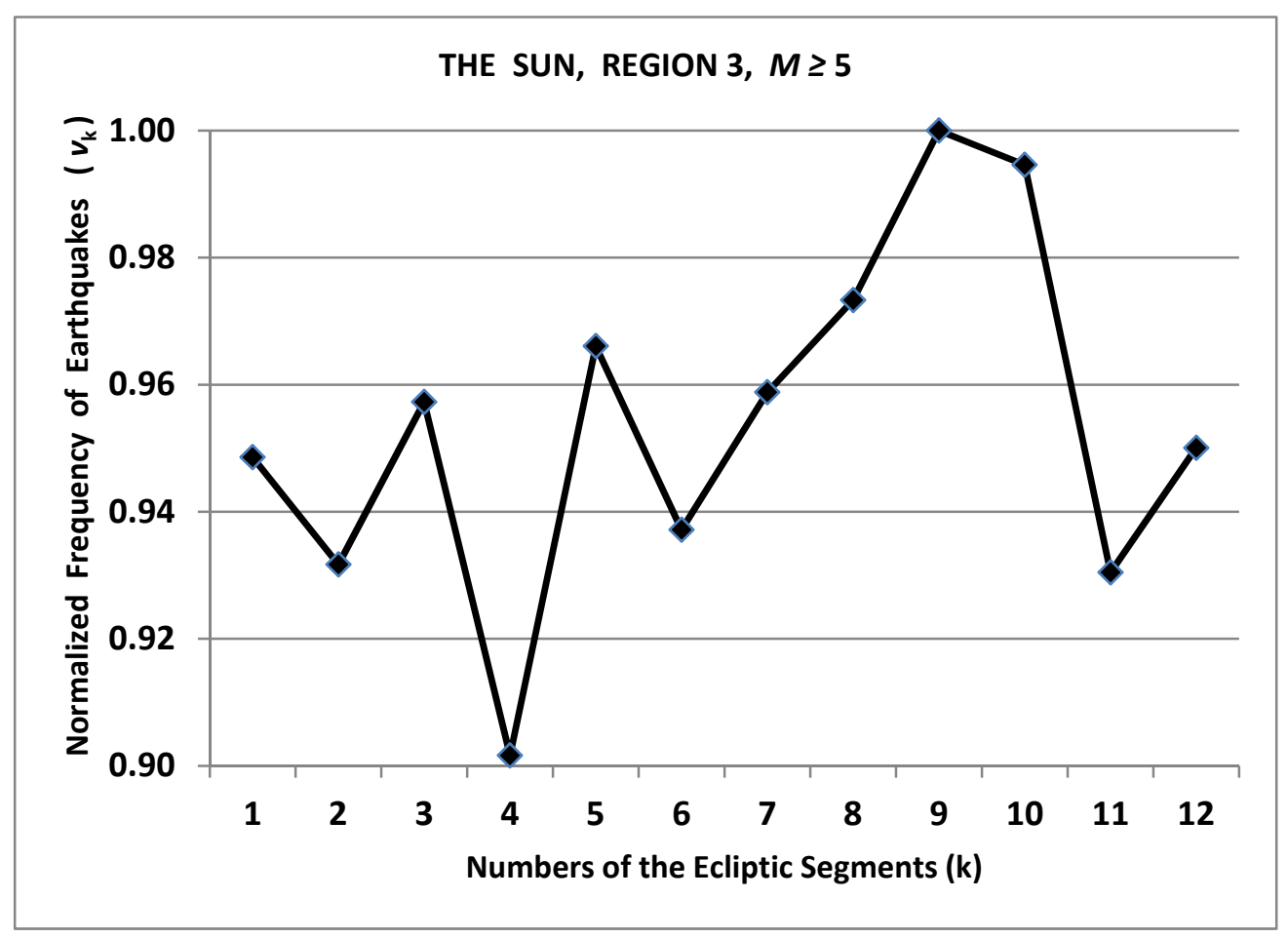

Figure 6. Region 3 - all terrestrial globe. The variations of the $v_{k}$ average earthquakes frequency in depending on the Sun position on the ecliptic during the period $1973-2009$, magnitude $M \geq 5$. The graph maximum normalized per unit

explanations to it are not present. Note that some community of the influences on seismic activity of different celestial bodies can be seen. This community is now best manifested itself in the fact that these influences depends on the celestial body ecliptic longitude. Therefore, in the opinion of the authors, it is necessary to search for the certain such reason which is common reason of the influences on seismic activity of different celestial bodies. Whether there is such common reason? We shall consider this problem within the framework of the classical (non-quantum) field theory.

According to the physical field theory concept, celestial bodies can act on seismic activity only by their physical fields. Neither gravity nor, especially, the electromagnetic fields of the planets can influence on terrestrial processes, because, according to the substantiation (Section 1), the intensity of these fields of the planets fall to an insignificant, negligible level at interplanetary distances. This repeatedly and reliably demonstrated in astrophysics. In accordance with the substantiation (Section 1), not only the gravitational and electromagnetic fields, but no other fields of planets and stars can not act on seismic activity, or rather, no other energy fields. Consequently, the planets and stars can influence on seismic activity only by their $F$ energy free fields. In addition, the influences of these $F$ fields of the planets and stars should be visible at the interplanetary and interstellar distances, respectively. Then, the very existence of the planets influences on seismic activity is evidence of the $F$ fields existence. The $F$ fields existence follows also from non-seismological experimental and observational data (Vasiliev, 2004 - 2012). Such fields are called the long-range action fields (LRA-fields) of the planets and stars (Vasiliev, 2008 - 2012). Problem of the LRA-fields existence are discussed in detail in the papers (Vasiliev, 2004, 2008a, 2008c, 2009b, 2012a, 2012b). The physical model of LRA-fields developed previously, as a logical consequence of the experimental and observational data (Vasilyev, 2004 - 2012). The LRA-fields physical model reveals the structure, some specific features and the origin conditions of LRA-fields. Like any physical field, LRA-field is a field-mediator in the transfer of actions at the distance. (It should not be confused LRA-field with "the long range action", which refers to the transfer of physics actions at the distance without intermediaries.) If the LRA-fields of the planets and stars act on seismic activity, then the LRA-field of the Sun (or Moon) and LRA-field of the Earth should also act on seismic activity. Consequently, the LRA-fields actions represent itself as the common reason of the influences on seismic activity of different celestial bodies. Let's see whether the actions of the celestial bodies LRA-fields can explain the factual specific properties of the 
celestial bodies influences on seismic activity.

In passing, we note that the influences of the planets on the Earth internal processes is not something exceptional. The planets act also on the Sun internal processes. Recently I. Charvatova has disclosed influences of the planets Меркурий, Venus, Earth, Mars to solar activity and solar-terrestrial phenomena (Charvatova, 2007, Dmitriev et al., 2009). And, I. Charvatova has revealed the role of the planets oppositions in the phenomena. According to the physical model of LRA-fields, just at the time moments of the planets oppositions relative to the Sun (and in special other instants) the abrupt flashes of the LRA-fields actions of the planets to the Sun occur with the possible long-lived aftereffect. The academic science starts to recognize influences of planets on the Sun interior processes. For example, as it is marked in the handbook (Dmitriev et al., 2009): "Physical origins of solar cycles are not known. They can be the internal property of the Sun as a star and its dynamo action as mostly believed now. Otherwise, planetary influences and interstellar causes could be involved. For example, it is believed sometimes that orbital rotation of giant planets (Jupiter, Saturn, Uranus and Neptune) is a natural source of the solar activity north-south asymmetries, and decadal and secular variations in the range of periods from $\sim 11$ years to 165 years" - the end of the citation.

\subsection{The Explanations of the Multiform Properties of the Celestial Bodies Actions on Seismic Activity from the Uniform Positions}

By virtue of said, within the framework of the classical field theory, from all fields of Mars, figures 1, 2, 3 show the actions of LRA-field of Mars only. Whereas for the Sun, figures 4, 5, 6 can show the influences not only of LRA-field of the Sun, but its other fields. Hence, to isolate the LRA-field action in the case of the Sun are more complex than in the case of Mars or other planets.

According to the physical model, $F_{S}$ LRA-field of the Sun or $F_{M}$ LRA-field of Mars (and of other planets) change along the whole length of the $\mathbf{R}_{\mathrm{E}}$ vector at $\psi$ rotational displacement of vector $\mathbf{R}_{\mathrm{E}}$ around of the Sun or Mars (and of other planets), respectively, and $\varphi=\psi$ (Section 2). Hence, according to the Mars displacement in the $\varphi$ angle on the ecliptic, its action on seismic activity should be changed, that is observed as in the graphs of Figures $1-3$ and in Lezdinsh's examinations. Analogously, the same is true for the Sun. At that, the Sun LRA-field action should be repeated with one-year period and to contribute to the detected year periodicity of seismic activity. (Because of the complex movements of Mars relatively the Earth, Mars has no constant period of its revolution on the ecliptic (at the sight to Mars from the Earth). The factual continuance of its revolution on the ecliptic varies from 542 up to 720 days for 1973 - 2009.) According to the physical model, LRA -fields of the Sun, Mars and other planets are different and differently depend on the $\psi$ angle. Consequently, the dependence on the $\varphi$ angle of their influences on seismic activity should be different also, that is conformed with the experimental data.

According to the physical model, LRA-field depend on external and internal movements of its source, the source structure and processes occurring in it. On the other hand, by the reciprocity principle, action of everyone LRA-field should depend on interior motions of the object of the action, from its structure and the processes taking place in it. The objects of the actions, in our case, are seismically subregions. The accumulation of earthquakes energy in these sub-regions are the result of complex internal processes of the Earth, varying from the subregion to subregion and occurring in different structural conditions. Therefore, the LRA-fields action of the Sun and planets on seismic activity, in its basic essence, should be different in the different subregions, that is brightly exhibited, as it is marked in the papers (Levin et al., 2005; Sasorova et al., 2006; Andreeva et al., 2009), in a reality concerning the actions of the Sun. This is seen in the graphs of the figures $1-6$ also concerning actions as the Sun and planet Mars. Hence and the Lezdinsh's metod should vary from the subregion to subregion with its special local developmental work depending on the local conditions. This is underlined by Lezdinsh also based on his practical experience of the short-term earthquake forecasting.

By virtue of the above, LRA-fields, generated by the Sun, stars, Earth, planets, Moon, carry in itself information about the interior processes of their sources. The changes of these interior processes, in particular, the structural reorganizations should be displayed in the changes of corresponding LRA-fields. Therefore, the monitoring of these fields is the kind of the monitoring of the internal processes of their sources. Here different prospects are unclosed and the comprehension of interrelationship and the certain unity of processes in our cosmos is looked through. We shall touch on here only those prospects that are close to the subject of this paper.

If the processes inside the Sun vary sharply, for example at the preparation of a high-power solar flare, its LRA-field should vary sharply. Thus, in the way of monitoring of the Sun LRA-field it is reasonable to search the precursors of solar flares, sunspots and solar weather. Since the LRA-field detector can indicate the direction to the LRA-field source (Smirnov et al., 2008), in principle, it is reasonable to develop the direction finding of the places where the LRA-field anomalies arise particularly with respect of LRA-field of the Earth. It is useful to not forget 
that we live in the "frying pan" in the form of the thin hard shell of the Earth under which there are the high-temperature interior processes of the Earth which are sometimes burst out through volcanoes. As you know, the high-precision gravimeters record the abrupt time gravity anomalies that indicate to abrupt movement of the large masses somewhere inside the Earth, but no one knows where. By virtue of the above, the mentioned drastic movement should generate the abrupt time anomalies of the Earth LRA-field. The direction finding of these abnormalities uncloses the prospects of the anomalies source place and time definition and the prospects of their coordination with forthcoming energy accumulation of volcanic eruptions and earthquakes. The same applies to the collisions of tectonic plates, preparing future earthquakes. The subcortical melt convection entrains tectonic plates. In the zone of the tectonic plates collisions - in the zone of future earthquake source, in result, the tectonic plates movements, the hot melt movements adjacent to the tectonic plates, the rock structure (the formation of small and medium-sized bed joint, substances metamorphism under high pressure, etc.) are changed. It should be displayed by beginnings of the smooth or sharp anomalies of the Earth LRA-field - by the earthquakes precursors, showing the locations of the future earthquakes by the direction finding. It looks like, the precursors in the form of sharp anomalies of the Earth LRA-field are registered by V. N. Smirnov in several cases for 2-10 days before the strong earthquakes (Smirnov et al., 2008). The precursors picked out, at first, by the detector directedness to the future earthquake sources, and secondly, by the specific amplitude and duration of the signal. To be sure, the continuous monitoring and direction finding are necessary with repeatedly confirmed by future earthquakes. Instead of the continuous monitoring, we have, unfortunately, the rare occasional short-time observations of the Earth LRA-field without direction finding.

The variations in the interior processes of the Sun (reflected by variations of solar activity) should lead to variations of the Sun LRA-field, which, in one's turn, should act on seismic activity. Hence, the Sun LRA-field should generate such correlation of solar and seismic activities which should be exhibited not only after the strong solar flare, but also at the stage of their preparation by the interior processes of the Sun. According to the statistical data, the correlation between solar and seismic activities was discovered (Section 2). In view of this, it is advisable to look for the indications of this correlation both after and before the solar flares.

According to the physical model (Vasiliev, 2008 - 2012), the one of the periods of the Sun LRA-field action is equal to solar day $T_{S U N}$. This cyclic recurrence, possibly in combination with other factors, should generate solar diurnal periodicity of earthquakes. But, since the Sun LRA-field actions on the earthquake preparation zones are different in different sub-regions, the expressiveness of solar - diurnal periodicity may vary between the sub-regions, as this is observed in practice (Sidorin, 2009). In addition, the Sun LRA-field has the brief abrupt flashes of its actions at sunrises, sunsets, the Sun culminations and at series of other time moments (Vasilief, 2008a, 2008b, 2009b). Therefore, the above-mentioned data of Ben-Menachem about the changes of seismicity at sunrises - sunsets cease to be surprising. Moreover, it is advisable to look for the seismicity abnormalities and at the other above-mentioned time moments series.

But, except for the $T_{S U N}$ period, earthquakes should have the period equal to $T_{S T}$ sidereal day. The matter is that LRA-fields have the following feature (Vasilief, 2008a, 2008b, 2009a, 2009b): celestial bodies and the Earth rotate around its own axis, but their LRA-fields do not rotate. Each of these LRA-fields depends on the non rotating polar angle coordinates associated with the center of the LRA-field source. LRA-fields form, as though, an invisible non-rotating (inertial) coordinate systems (Vasilief, 2009b). Therefore, each of the earthquake source, rotating around of the Earth axis, cyclically passes through the Earth LRA-field for every revolution. By definition, the period of the rotation is $T_{S T}$ sidereal day. Consequently, the Earth LRA-field acts on the earthquake preparation zones with the $T_{S T}$ period and acts unevenly within the sidereal day. Hence, we should expect the diurnal earthquakes periodicity with the $T_{S T}$ period and nonuniformity of the earthquakes distribution inside the sidereal day. The external global constant direction LRA-field has the same $T_{S T}$ period of the actions on terrestrial objects (Vasilief, 2009a). Figure 7 shows a graph of the frequency changes of earthquakes with $M \geq 5$ in Greece for the period 1950 - 2008 depending on the local sidereal time. The uneven distribution of earthquakes on sidereal time is really presented and reaches $42 \%$ (Figure 7 ). Hence, the earthquakes frequency really depends on the sidereal time. The data source was the Greek earthquake catalog available online www. gein.noa.gr / services / cat.html. The number of earthquakes occurred in $0-2,2-4,4-6, \ldots, 22-24$ clocks of the local sidereal time was summed for 1950 - 2008. The diagram of these sums is shown on the Figure 7. As well as earlier, the maximum of the diagram is normalized to unity. At calculations of the of the Figure 7 diagram it was taken into account about 500 earthquakes. Here the statistics and $P_{M A X}=P_{M I N}=0.98$ probabilities are not too the strong. It is expedient to strengthen them having prolonged research. 


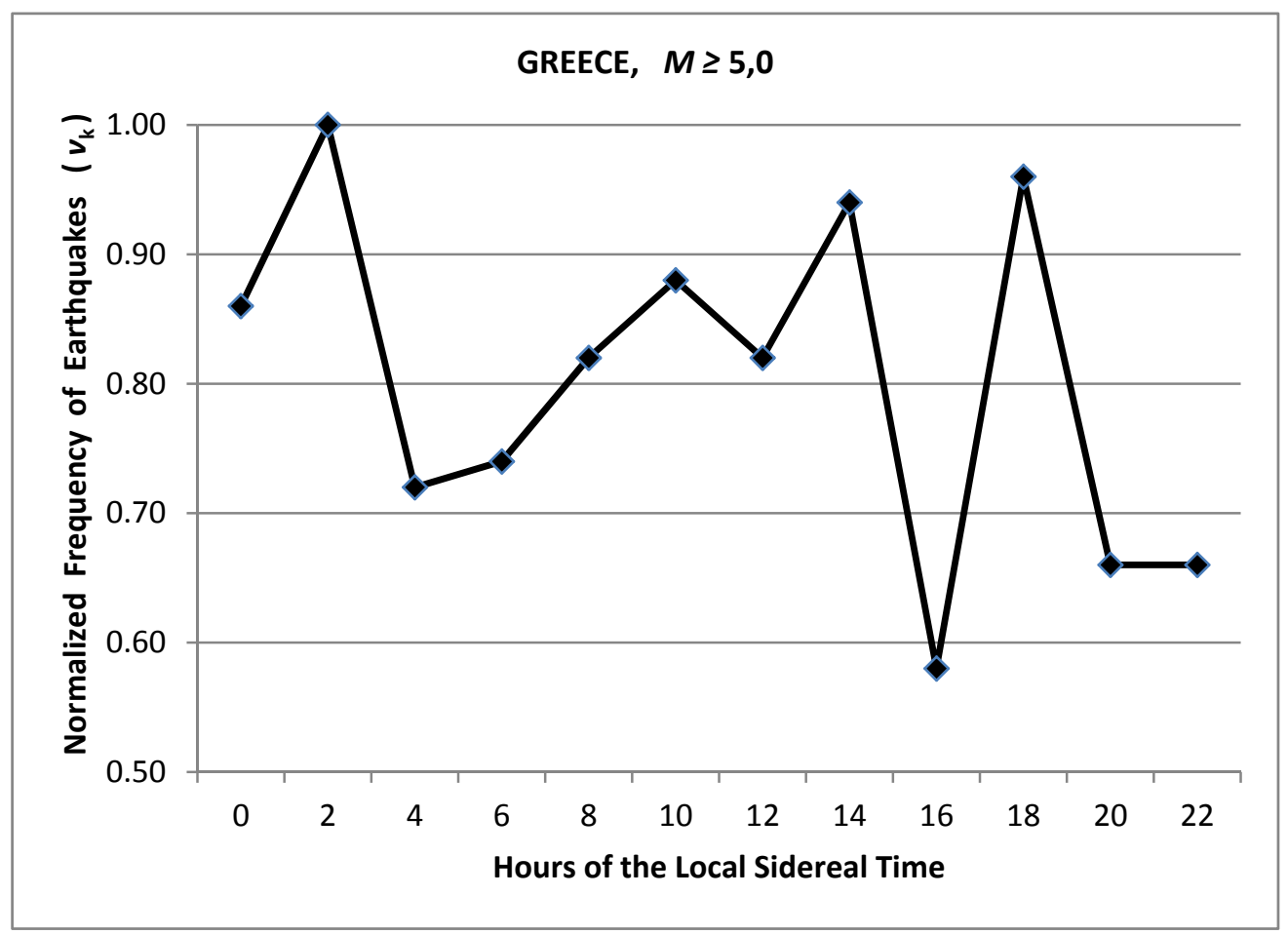

Figure 7. Greece. The variations of the $v_{k}$ average earthquakes frequency in depending on the local sidereal time during the period $1950-2008$, magnitude $M \geq 5$. For each two-hour interval of $0-2,2-4,4-6, \ldots, 22-24$ hours of the local sidereal time the number of earthquakes that have occurred in this interval summed up for the entire period 1950 - 2008. The graph maximum normalized per unit

Consequently, seismic activity reacts to actions of LRA-fields in the same way as the Shnoll histograms (Shnol, 2009; Vasilief, 2009a, 2009b), that is, under the influence of LRA-fields seismic activity gets the year cycle (the influence of the Sun) and two diurnal cycles with the periods equal to the $T_{S U N}$ solar day (the influence of the Sun) and the $T_{S T}$ sidereal day (the influences of the Earth LRA-field and external global field). Besides seismic activity, as well as the Smirnov detector, reacts to sunrises, sunsets, to the influence of the planets and stars. In other words, seismic activity is the peculiar but complex natural detector of LRA-fields.

It is interesting to note that, in calculating the $N_{k}$ numbers, the input data - numbers $N_{k j}$ - can be replaced by the results of their smoothing on the $j$ index (that is, on time) by the third-degree polynomials. It was found that under such replacement the $N_{k}$ numbers change only in the third and fourth signs in our experiments, and the 1 - 6 figures graphics are practically unchanged. So the long-term accumulation of the earthquakes numbers for each $E_{\mathrm{k}}$ segment well filtered out the short-term variations of the earthquakes frequency. These polynomials can be useful for analytical calculations and analysis by formulas (for example, at the regularization of unstable Fourier transform of the time earthquakes series). These third-degree polynomials are variable on time functions. Seemingly, the indicated smoothing, rejecting transient flashes, indicates to a relatively slow changes on time of the earthquakes origin conditions under the celestial bodies actions (at least, under the Sun and Mars actions). These changes may be the result of gradual changes of the tectonic conditions (in seismoactive subregions) and/or the celestial bodies LRA-fields.

As it mentioned above, the physical nature of LRA-fields is now unknown. The mechanism of their actions on seismic activity is not clear. According to seismology, earthquakes happen as a result of collision of large plates of the Earth's crust floating on the underlying melt, that is, as a result of the internal motions of the Earth. The tensions increase in the collision zone inside rock formation. At the moment, when these tensions begin to exceed ultimate strength of the rock formation, the earthquake arises from it. Thus, in principle, the influence to origin of earthquakes can happen in the two-fold way: by actions to the Earth internal motions and by changes of the ultimate strength of rock formation, for example, due to change of structural peculiarities of the rock formation. Probably, the celestial bodies directly influence to motions on the Earth or to structures of the Earth substances. There are some experimental testimonies for the both assumptions. 
At first, the experiment shows the direct influence of the celestial bodies to motions on the Earth - influences to motions within the Smirnov detector and to motions of torsion balances. Second, there are the experimental testimonies on directly influence of celestial bodies to structure, and, therefore, to the ultimate strength of the Earth substances. So, the researchers of the Siberian Branch of Russian Academy of Sciences found (Eganova, 2005) that the far from us collision of the Jupiter with the comet SL-9 generated, however, on the Earth the contrasting changes of the behaviour of mechanical and physical-chemical systems, over which the long-term calendar scientific observations were carried out. The researchers found the following (Eganova, 2005): "The rotational displacement of the asymmetric torsion balance, which occurred over the entire period of the catastrophic event on Jupiter in July 1994, lasted until 21 October, after which the torsion balance back to its normal state with daily torsional vibrations, and, the return act had occurred without any further relaxational vibrations. ... Particular interest is the reaction of an old English device - of stormglass: the large ampoule contains the specially prepared the complex mixture, which combines great number of substances: water, camphor, ammonia, nitrate, and alcohol. Mariners had used this device as a predictor of the weather. After those events on Jupiter, a great layer of crystals had arisen in the stormglass and that layer was not eventually dissolved (as it usually happens), it is compacted and stored until now, that is, more than 10 years .... Moreover, in one stormglass which was put in the thermostat $\left(35.1^{\circ} \mathrm{C}\right.$ ), this layer had disappeared (note that a stormglass operates in a thermostat, in principle, as usually), but when after few years the stormglass was removed out from the thermostat, the same layer was recovered (!) with time." - the end of citation. Recently, using special spectroscopy, Zubow and his colleagues discovered the changes of supramolecular structures of many terrestrial objects under the influences of the Sun, Moon and planets (Zubow et al., 2010-2012; Vasiliev, 2010). These objects are: liquid and solid media, in particular, solutions and water. Zubow and his colleagues proposed the physical-chemical model of the conversion of these changes within earthquake source in initialization of the earthquake (Zubov et al., 2012a). The authors can not agree with the explanations of reasons of the supramolecular structures changes given in papers (Zubov et al., 2010-2012), but believes that their experimental part deserves of attention and development to a systematic scientific observations. Similar observations are useful to accomplish systematically and in relation to the rock formation in the interests of seismology.

At last, we note that in the future, it is not excluded, it will necessary to take into account changes of the stars positions relative to the local horizon plane (for example, in the method of Lezdinsh). These changes generate abrupt flashes of the celestial bodies actions at their specific positions relative to the local horizon plane, in particular, at their transits (Vasiliev, 2008b, 2009b, 2009d). Influence of the stars on terrestrial objects may seem improbable.

Nevertheless, in the Smirnov detector, the gyroscope angular velocity varies under actions of the nearby stars, for example, of the star Sirius, the star $\xi$ Eri (HP 15197), the star $\alpha$ For (HP 14679A), and of distant galaxies, for example, of Andromeda nebula and the galaxy NGC 1344 (Smirnov et al., 2009; Pancheliga, 2012). This happens at the moments of the stars and galaxies upper transits without applications of focusing systems. Moreover, the stars and galaxies actions to the gyroscope were not insignificant. They were quite comparable with the planets actions. (On the signal attenuation of the celestial bodies LRA-fields with distance, please, see the paper (Vasiliev, 2012a).) Zubow and his collaborators discovered the following: a) the contrasting changes of supramolecular structures at the moments of the upper transits of the Galaxy center (Zubow et al., 2012b); b) the dynamic viscosity dominant changes and changes of supramolecular structures at the moments of the upper and lower transits of the star cluster M34 (Zubow et al., 2011b); c) the dynamic viscosity changes at the moments of the transits of the galaxy VIRGOHI21 in opposition to the Sun (Zubov et al., 2011b). (In these papers the transits are termed as "passing through the plane of the gravitational resonance")

\section{Summary}

In this paper the influences of the planet Mars and the Sun on seismic activity in the subregion of the U.S. west coast and part of Mexico, in the subregion of Japan and in the world as a whole are confirmed statistically reliably (Section 3). The common properties and certain differences of the actions of Mars and the Sun on seismic activity are marked. In particular, the obtained statistical data confirm that, in some cases, the Mars actions on seismic activity appear more strongly of the Sun actions (Sections 2 and 3). Our statistical data, together with data of other researchers (Section 2), lead to the conclusion that the actions of the Sun, planets, and even stars on seismic activity is the fact. It should have an explanation. In respect to the Sun actions on seismic activity, many hypotheses were putted forward but the reasons of the actions unambiguously were not determined and remain vague (Section 2). In respect to the planets actions there are no even hypothetical physical explanations. The influence of the planets on the Earth interior processes is not something exceptional. The planets act also on the 
Sun interior processes (Section 4).

According to Section 4, within the framework of the classical (non-quantum) field theory concept and by virtue of the substantiation (Section 1), the actions of solar system planets and stars on seismic activity can be carried out only by means of them Long-Range Action fields (LRA-fields). The Sun, Moon and Earth have LRA-fields also and also can act on seismic activity by means of their LRA-fields (but not only). Therefore the LRA-fields actions are the reason of the influences on seismic activity common for different celestial bodies and the Earth. The physical model of LRA-fields developed previously (Vasiliev, 2004 - 2012). It reveals the structure, specific properties and the origin conditions of LRA-fields. According to our (Sections 3 and 4) and other (Section 2) examinations, the celestial bodies influences on seismic activity have a variety of characteristic properties which are important both for the practical purposes of the earthquakes forecasting and for understanding of the relation of seismic activity and celestial bodies. These properties should have the explanations. The LRA-fields physical model allows to explain and predicts the following (Section 4): the dependence of the celestial bodies influences on seismic activity from their ecliptic longitude; the distinctions of this dependence for different celestial bodies; the year cycle of seismic activity and non-uniformity of the earthquakes distribution on months; the cycles of seismic activity with periods equal to the planets revolution periods on the ecliptic; non-uniformity of the earthquakes distribution on local solar time; non-uniformity of the earthquakes distribution on local sidereal time; the distinctions of the same celestial body influences in different seismoactive subregions; the seismic and solar activity relation; the different expressiveness of the diurnal seismic cycle in different subregions; the changes of seismic activity at sunrises and sunsets; the earthquake precursors appearance in the form of the Earth LRA-field anomalies.

The physical model predicts also the precursors of solar flares and the solar weather changes in the shape of the Sun LRA-field anomalies (Section 4). Monitoring of LRA-fields opens new perspectives of the seismic activity and solar weather forecasting, new perspectives of tracking of the Earth and celestial bodies interior processes changes since the variations of LRA-fields reflect the changes of the internal processes of their sources. There is the opportunity for the direction finding of the sources of the LRA-fields anomalies that, in principle, indicates to the perspectives to determine both the time and place of future earthquakes, volcanic eruptions and solar flares by means of this direction findings (Section 4). Thus, the use of LRA-fields starts to acquire practical significance.

Hypothetically, the actions of LRA-fields on the earthquakes origin can occur by the two-fold way: by actions on the interior motions of the Earth and by changes of the rock formation ultimate strength. There are some experimental testimonies in favour of the both assumptions (Section 4).

Necessary to emphasize the following. The existence of LRA-fields generates a certain correlation of seismic activity with the celestial bodies configuration. But also there should be a correlation between seismic activity with a variety of other factors (the electromagnetic, gravitational, mechanical, chemical, etc. actions). Therefore, the only correct way to use the correlation with the celestial bodies configuration will consist, according to opinion of the authors, in its complexation with other correlations. For example, the method of the short-term forecast of the place, time and magnitude of strong earthquakes (Lezdinsh, Section 2) is effective in practice because it uses not only the correlations with the positions of the planets, Sun, Moon relative to the Earth and relative to the local horizon plane but else uses the correlations with the seismological factors. It has been empirically unequivocally established that if in Lezdinsh's method to not use the correlation either with seismological factors or with astronomical factors, the method becomes inefficient. The complex multifactorial influences on seismic activity forces to prefer the epoch superposition method for detection of the influence of the separately taken factor as it is made in this paper.

The authors of this paper had no at their disposal of computer processing aids of the earthquakes time series. Therefore data about the tens thousand earthquakes were processed almost manually. It was a huge, almost back-breaking toil. Moreover, the work done is several times greater than its parts contained in this paper. But the authors went on it in order to statistically reliable answer to the fundamental question: is there or not the influence of the planets on seismic activity, and how this influence is compared to the Sun influence. Now the key positive answer is obtained. Now it is necessary to have begun to investigate the planets actions on seismic activity with all power of the seismological hardware and computer processing aids.

\section{Acknowledgements}

The authors thank seismologists the member-correspondent of the Russian Academy of Science (RAS) A. V. Nikolaev, Professor E. V. Sasorova, the President of the European Seismological Commission A. D. Zavyalov, the specialist on a solar weather Dr. A. D. Dmitriev, the specialist on a geoelectrics professor M. N. Yudin and the author of the innovational method of short-term forecast of the strong earthquakes place, time and magnitude A. 
Ya. Lezdinsh for the valuable consultations, useful advices and discussions. The authors are grateful to A. V. Nikolaev for initialization of the statistical examinations of the planets influences on seismic activity and to A. Ya. Lezdinsh for providing the details of his research.

\section{References}

Andreeva, M. Yu., Sasorova, Ye. V., \& Levin, B. V. (2009). The features of within-year earthquake distributions in the Kuril region. Tihooleanskaya geologia, 28, 5. 85-95 (in Russian).

Charvátová, I. (2007). The prominent 1.6-year periodicity in solar motion due to the inner planets. Ann. Geophys., 25, 1227-1232. Retrieve from http://www.ann-geophys.net/25/1227/2007/angeo-25-1227-2007.pdf.

Dmitriev, A. V., Suvorova, A. V., \& Veselovsky I. S. (2009). Statistical characteristics of the heliospheric plasma and magnetic fields at the Earth's orbit during four solar cyclea 20-23. In: Handbook on Solar Wind: Effects, Dynamics and Interactions, Ed. Hans E. Johannson, 2009, chapter 2, 81-144. New York: NOVA Science Publishers, Inc.

Eganova, I. A. (2005). Terra incognita, unclosed by Kozyrev N. A. 5-n the Siberian interdisciplinary conference "Mathematical problems of the physics of space - time of the composite systems", 2004, Library of conference, issue 2, 249-271. Novosibirsk, publishing house of the Siberian Deppartment of the Russian Academy of Sciences, editor Lavrentjev M.M. (in Russian)

Khain, V. E., \& Khalilov, E. N. (2008). About possible influence of solar activity on seismic and volcanic activities: long-term forecast. Science Without Borders, Transactions of the International Academy of Science $H \& E, \quad 3,2007 / 2008, \quad 316-334, \quad S W B$, Innsbruck, 2008. Retrieved from http://book-ias-he.org/volume3.pdf

Kiladze, R. I., Kachakhidze, M. K., Kachakhidze, N. K., Kukhnanidze, V. D., \& Ramishvili, G. T. (2005). Seaching for possible connections between strong earthquakes and astronomic phenomena on the example of seismically active region in Caucasus. Vulkanologia i seismologia, No. 3, May-June, 78-84 (in Russian).

Levin, B. V., Sasorova, E. V., Zhuravlev, S. A. (2005). The intraannual reiteration of the seismic process activation for the Pacific region. Docladu Academii Nauk, 403(4), 1-7. (in Russian)

Lezdinsh, A. Ya. (2008). Astroseismology, The Earth Planet System, Proceedings of XVI-th Scientific Seminar (221-225). Moscow State University, Lenand, Moscow (in Russian).

Magnitsky, V. A. (2006). The internal structure and the physics of the Earth (p. 390). Book, Nauka, Moscow (in Russian).

Panchelyuga, V. A. (2012) The Smirnov detector: Recording of the actions from distant astrophysical objects. Metaphysics, 2(4), 67-80, Retrieved from http://www.intelros.ru/pdf/metafizika/2012_02-04/04.pdf (in Russian).

Sadeh, D., \& Meidav, M. (1972). Periodisities in seismic response caused by pulsar CP1133. Nature, 240, November 17.

Sasorova, E. V., \& Zhuravlev, S. A. (2006). The peculiar properties of the within-year periodicity for seismic event distributions for some Pacific regions and astronomical factors. S. Mukherjee (Ed.), "Earthquake Prediction”. Earthquake Prediction (9-20). Boston: Brill Leiden.

Shnoll, Simon E. (2009). Cosmic physical facrors in random processes. Svenska fysikarkivet, Stockholm (388 p., in Russian). Retrieved from http://www.ptep-online.com/index_files/books.html

Sidorin, A. Ya. (2009). The diurnal periodicity of earthquakes and its seasonal changes. Seismicheskie priboru, 45(4), 69-84 (in Russian).

Smirnov, V. N., Egorov, N. V., \& Panchelyuga, V. A. (2009). On recording of the action of the nonelectromagnetic nature from the far removed astrophysical objects. In international conference "Physical interpretations of the relativity theory", on July, 6-9, Moscow State Technical University of a name of N.E. Bauman, Moscow (in Russian).

Smirnov, V. N., Egorov, N. V., \& Shchedrin, S. I. (2008). A New Detector for Perturbations in Gravitational Field. Progress in Physics, 2, 129-133.

Vasiliev, S. A. (2004). The problem of the construction of physics of the non-material world and its value for all of us. Moscow: Christian publishing house, $(82$ p.) (in Russian). Retrieved from 
Www.nonmaterial.pochta.ru or www.nonmaterial.narod.ru .

Vasiliev, S. A. (2008a). On some field of the Earth in view of its internal motions (in Russian). Degassing of the Earth: geodynamics, geofluids, oil, gas, and their parameters, Proceedings of All-Russian Conference, Moscow, April, 22-25, 576-579. Moscow: Publishing House GEOS.

Vasiliev, S. A. (2008b). The comparison of the experimental and some long-term observation data on the two-component field of the Earth. The Earth Planet System, Proceedings of XVI-th Scientific Seminar, Moscow State University, The monography, p.120-141. Moscow: Book house "LIBROCOM", (in Russian). Retrieved from www.nonmaterial.pochta.ru or www.nonmaterial.narod.ru .

Vasiliev, S. A. (2008c). The answers to the questions and objections frequently arising in scientific discussions. The Earth Planet System, Proceedings of XVI-th Scientific Seminar, Moscow State University, The monography, p.200-215. Moscow: Book house "LIBROCOM", (in Russian). Retrieved from www.nonmaterial.pochta.ru or www.nonmaterial.narod.ru .

Vasiliev, S. A. (2009a). On the Physical Model of the Phenomena Registered in the Experiments by Shnoll's Group and Smirnov's Group. Progress in Physics, 2, 29-43. Retrieved from http://www.ptep-online.com/index_files/2009/PP-17-07.PDF.

Vasiliev, S. A. (2009b). Whether there is the long-range action fields of the Earth and celestial bodies? - the brief review of the results of examinations. The Earth Planet System, Proceedings of XVII-th Scientific Seminar, 15 years to the interdisciplinary scientific seminar, Moscow State University, Moscow, The monography, p.72-104. Moscow, LENAND, (in Russian). Retrieved from www.nonmaterial.pochta.ru or www.nonmaterial.narod.ru .

Vasiliev, S. A. (2009c). On the opportunities, problems and value of the construction of physics of the non-material world. The Earth Planet System, Proceedings of XVII-th Scientific Seminar, 15 years to the interdisciplinary scientific seminar, Moscow State University, Moscow, The monography, p.117-150. Moscow, LENAND, (in Russian). Retrieved from www.nonmaterial.pochta.ru or www.nonmaterial.narod.ru .

Vasiliev, S. A. (2009d). Whether there is the paradoxical long-range action? The physical theory and experiment. Spravochnic. Ingenernuy jurnal, 9, 55-64 (in Russian).

Vasiliev, S. A. (2010). The problems and the example of searching and experimental researches of actions of the sector long-range action fields of the Earth and celestial bodies on the physicochemical parameters of terrestrial objects. The Earth Planet System, Proceedings of XVIII-th Scientific Seminar, 300 years from M.V. Lomonosov's birthday, 1711 - 2011, Moscow State University, p.190-214. Moscow: Book house "LIBROCOM", (in Russian). Retrieved from www.nonmaterial.pochta.ru or www.nonmaterial.narod.ru .

Vasiliev, S. A. (2012a). The classical concept of the existence of the long-range action fields. Applied Physics Research, 4 (1), 167-177. http://dx.doi.org/10.5539/apr.v4n1p167.

Vasiliev, S. A. (2012b). Basic Physical Properties of the Physical Non-material World Objects. Applied Physics Research, vol. 4 (2), 175 - 189. http://dx.doi.org/10.5539/apr.v4n2p175.

Vikulin, A. V. (2003). Physics of wave seismic process (150 p.). KPGU, Petropavlovsk-Kamchatski, Russia, Editor the member-correspondent of the Russian Academy of Science (RAS) A. V. Nikolaev, (in Russian). Retrieved from http://www.kscnet.ru/ivs/monograph/vikulin .

Wiemer, S. (2000). Earthquake Statistics and Earthquake Prediction Research. Institute of Geophysics; Zürich, Switzerland. Retrieved from http://www.earthquake.ethz.ch/education/NDK/NDK

Yigiter, A. (2012). Change Point Analysis in Earthquake Data, Earthquake Research and Analysis - Statistical Studies, Observations and Planning, Sebastiano D'Amico, (Ed.) ISBN: 978-953-51-0134-5, InTech, retrieved from http://www.intechopen.com/books/earthquake-research-and-analysis-statisticalstudies-observations-and-pla nning/change-point-analysis-in-earthquake-data .

Zubow, K., Zubow, A. V., \& Zubow, V. A. (2011a). Scanning of the Sun and other celestial bodies with help of gravitation spectroscopy. Optic and Photonics Journal, 1, 15-23. http://dx.doi.org/10.4236/opj.2011.12004

Zubow, K., Zubow, A. V., \& Zubow, V. A. (2012b). Experimental Methods for the Determination of the Super Light Velocities of the Gravitation. Nature, Structure and Properties of Gravitation Waves. Horizons in World Physics, 277. 
Zubow, K., Zubow, A. V., \& Zubow, V. A. (2011b). The Phenomenon of Planets' Influence on the Long-Range Order in Polyethylene glycol and Its Dynamic Viscosity. Research and Reviews in Polymer, 2(2), (India).

Zubow, K., Zubow, A.V., \& Zubow, V. A. (2012a). Water Clusters Ensemble as a Interface to Structure of the Epicenter and the Earthquake Mechanism on the Jawa Island ( $8^{\circ} 73^{\prime}$ s. $112^{\circ} 36^{\prime}$ e.). J. of Phys. Chem., 7(3), 87-95.

Zubow, K. V., Zubow, A. V., \& Zubow, V. A. (2010). Ensemble of Clusters - New Form of Molecular Matter, Risks and Chances. Zubow Equations. Advances in Chemistry Research, 5, 107-145. Book, Editor J.C. Taylor. New York, NY: Nova Science Publishers, Inc. Retrieved from https://www.novapublishers.com/catalog/product_info.php?products_id=27769 